\title{
Simulated Power Spectra of Solar-Type Oscillations Driven by an Elementary Excitation Source
}

\author{
I. W. Roxburgh and S. V. Vorontsov ${ }^{1}$ \\ Astronomy Unit, Queen Mary and Westfield College, Mile End Road, \\ London $\mathrm{E} 14 \mathrm{NS}, \mathrm{UK}$
}

\begin{abstract}
We consider the synthetic power spectra of multi-mode solartype acoustic oscillations in stars, computed within a general framework of the linear response of the dynamical system to an arbitrary excitation force which is decomposed in spherical harmonics in space and harmonic functions in time. We discuss possible signatures in the observational data which might be used as diagnostics of the excitation source.
\end{abstract}

With recent progress in solar seismology, there is an increasing amount of signatures in the observational data which contain valuable information about the excitation source - the high-frequency "pseudo-modes" (Libbrecht 1988; see Kumar 1994 for a recent theoretical discussion) and line asymmetries at intermediate frequencies in the observational power spectra (Duvall et al. 1993; for theoretical discussion, see also Gabriel 1992). The proper interpretation of these observations will hopefully lead to an improvement in our general understanding of the turbulent convection which is responsible for the excitation of solar oscillations (see Goldreich et al. 1994 for a recent discussion). In this contribution, we consider similar observational information which might become available from the seismology of distant stars.

Our analysis is based on a simple asymptotic theory of the high-frequency acoustic oscillations, extended to include an elementary excitation source (Roxburgh \& Vorontsov 1995). We use the adiabatic approximation and simulate the energy sink in the dynamical system by allowing free outgoing wave as an upper boundary condition imposed at some level in the upper atmosphere.

The resonant frequencies in the power spectra are described by the equation

$$
\int_{r_{1}}^{R}\left[\frac{1}{c^{2}}-\frac{(\ell+1 / 2)^{2}}{\omega^{2} r^{2}}\right]^{1 / 2} d r \simeq \pi \frac{n+\hat{\alpha}(\omega)}{\omega}
$$

where the surface phase shift $\hat{\alpha}(\omega)$ is determined by the atmospheric structure and source location. This frequency equation extends the standard asymptotic eigenfrequency equation to include the high-frequency "pseudo-modes" and the effects of the simple excitation source. The "large frequency separation", the

\footnotetext{
${ }^{1}$ Institute of Physics of the Earth, Moscow 123810, Russia (permanent address)
} 


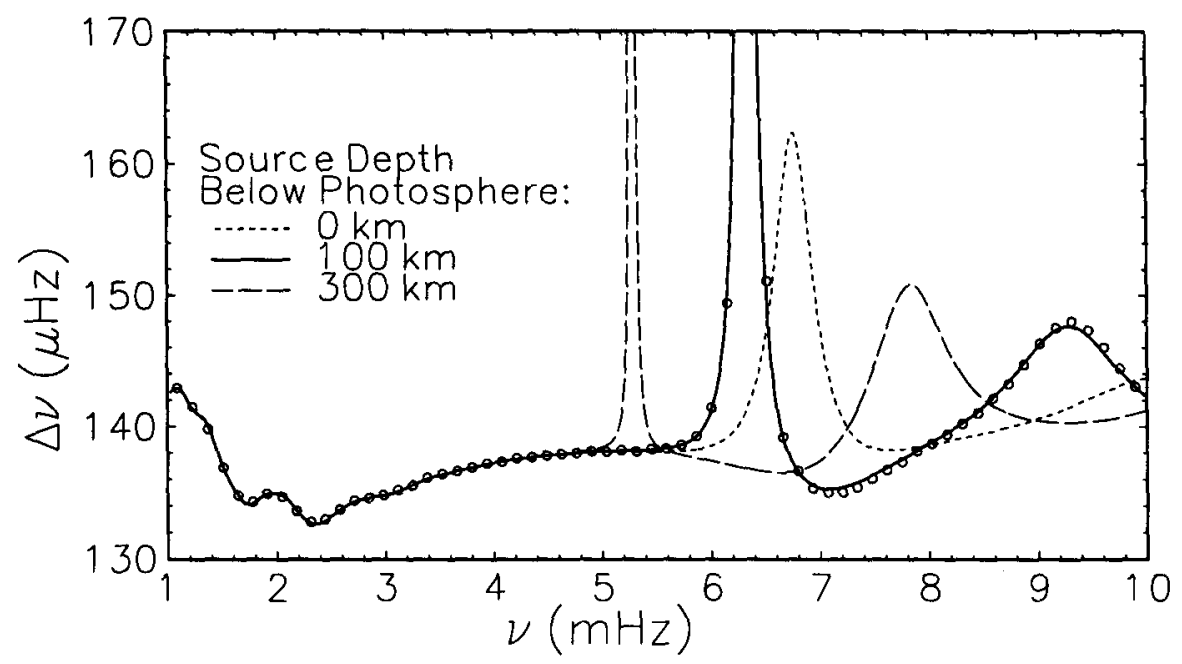

Figure 1. Large frequency separations computed with a dipole source.

measurable quantity in stellar seismology, is

$$
\Delta \nu=\nu_{n+1, \ell}-\nu_{n, \ell} \simeq \frac{1}{2 \pi}\left(\frac{\partial \omega}{\partial n}\right)_{\ell} \simeq\left[2 \int_{0}^{R} \frac{d r}{c}-2 \frac{d(\pi \hat{\alpha})}{d \omega}\right]^{-1}
$$

The "large frequency separations" computed for a solar model are shown in Fig.1. A sharp maximum in $\Delta \nu(\nu)$ is clearly seen at intermediate frequencies between the trapped modes and the high-frequency "pseudo-modes"; the position of this maximum is sensitive to the location of the excitation source.

The line profiles in the simulated power spectra also show a quite high sensitivity to the source position and to the relative strength of dipole and monopole components of the excitation. Observational data of exceptionally high quality are needed, however, for measuring the signatures in the power spectra which might be used for the diagnostics of the excitation source; more theoretical work is also needed to employ the diagnostic capability of these measurements, if they become available.

\section{References}

Duvall, T. L., Jr., Jefferies, S. M., Harvey, J. W., Osaki, Y., \& Pomerantz, M. A. 1993, ApJ, 410,829

Gabriel, M. 1992, A\&A, 265, 771

Goldreich, P., Murray, N., \& Kumar, P. 1994, ApJ, 424, 466

Kumar, P. 1994, ApJ, 428, 827

Libbrecht, K. G. 1988, ApJ, 334, 510

Roxburgh, I. W., \& Vorontsov, S. V. 1995, MNRAS, 272, 850 